\title{
Video Article \\ Osteopathic Manipulative Treatment as a Useful Adjunctive Tool for Pneumonia
}

\author{
Sheldon Yao ${ }^{1}$, John Hassani ${ }^{1}$, Martin Gagne ${ }^{1}$, Gebe George ${ }^{1}$, Wolfgang Gilliar ${ }^{1}$ \\ ${ }^{1}$ Department of Osteopathic Manipulative Medicine, New York Institute of Technology College of Osteopathic Medicine
}

Correspondence to: Martin Gagne at mgagne@nyit.edu

URL: https://www.jove.com/video/50687

DOI: doi: $10.3791 / 50687$

Keywords: Medicine, Issue 87, Pneumonia, osteopathic manipulative medicine (OMM) and techniques (OMT), lymphatic, rib raising, thoracic pump, muscle energy, doming diaphragm, alternative treatment

Date Published: 5/6/2014

Citation: Yao, S., Hassani, J., Gagne, M., George, G., Gilliar, W. Osteopathic Manipulative Treatment as a Useful Adjunctive Tool for Pneumonia. J. Vis. Exp. (87), e50687, doi:10.3791/50687 (2014).

\section{Abstract}

Pneumonia, the inflammatory state of lung tissue primarily due to microbial infection, claimed 52,306 lives in the United States in $2007^{1}$ and resulted in the hospitalization of 1.1 million patients ${ }^{2}$. With an average length of in-patient hospital stay of five days ${ }^{2}$, pneumonia and influenza comprise significant financial burden costing the United States $\$ 40.2$ billion in $2005^{3}$. Under the current Infectious Disease Society of America/ American Thoracic Society guidelines, standard-of-care recommendations include the rapid administration of an appropriate antibiotic regiment, fluid replacement, and ventilation (if necessary). Non-standard therapies include the use of corticosteroids and statins; however, these therapies lack conclusive supporting evidence ${ }^{4}$. (Figure 1 )

Osteopathic Manipulative Treatment (OMT) is a cost-effective adjunctive treatment of pneumonia that has been shown to reduce patients' length of hospital stay, duration of intravenous antibiotics, and incidence of respiratory failure or death when compared to subjects who received conventional care alone ${ }^{5}$. The use of manual manipulation techniques for pneumonia was first recorded as early as the Spanish influenza pandemic of 1918 , when patients treated with standard medical care had an estimated mortality rate of $33 \%$, compared to a $10 \%$ mortality rate in patients treated by osteopathic physicians ${ }^{6}$. When applied to the management of pneumonia, manual manipulation techniques bolster lymphatic flow, respiratory function, and immunological defense by targeting anatomical structures involved in the these systems $\mathrm{s}^{7,8,9,10}$.

The objective of this review video-article is three-fold: a) summarize the findings of randomized controlled studies on the efficacy of OMT in adult patients with diagnosed pneumonia, b) demonstrate established protocols utilized by osteopathic physicians treating pneumonia, c) elucidate the physiological mechanisms behind manual manipulation of the respiratory and lymphatic systems. Specifically, we will discuss and demonstrate four routine techniques that address autonomics, lymph drainage, and rib cage mobility: 1) Rib Raising, 2) Thoracic Pump, 3) Doming of the Thoracic Diaphragm, and 4) Muscle Energy for Rib $1^{5,11}$

\section{Video Link}

The video component of this article can be found at https://www.jove.com/video/50687/

\section{Introduction}

Children, elderly individuals, and immunocompromised patients are especially susceptible to pneumonia. Patients may present with fever, cough, dyspnea, tachypnea, tachycardia, sputum production, pleuritic chest pain, nausea, vomiting, diarrhea, and fatigue ${ }^{4}$. Clinical chest examination may reveal dullness to percussion and crackles upon auscultation over the affected lung ${ }^{12}$. Laboratory results may show a leukocytosis with leftshift, as well as an increase in erythrocyte sedimentation rate and C-reactive protein ${ }^{4}$. Tests to elucidate the specific causative microbe include the urine antigen test, polymerase chain reaction, sputum analysis, and blood culture ${ }^{4}$. Finally, radiographic imaging, which serves as the gold standard for diagnosis, may display consolidation or inflammatory changes over the various lobes of the lungs ${ }^{12}$. While no specific algorithm or constellation of symptoms have consistently proven to establish the clinical diagnosis of pneumonia, temperature $>100^{\circ} \mathrm{F}$ and abnormal breath sounds (rales or rhonchi) are generally accepted as criteria for ordering a chest x-ray or sputum sample ${ }^{13}$. Additionally, the Pneumonia Severity Index (PSI) and CURB-65 criteria have been proven to accurately predict mortality in pneumonia patients. ${ }^{14,15}$

Once the diagnosis of pneumonia has been confirmed, traditional standard-of-care management should be administered. For patients in a stable condition, osteopathic evaluation and adjunctive treatment may be beneficial and should be considered.

In addition to being fully-licensed to practice conventional medicine, osteopathic physicians utilize a multi-step holistic approach to diagnosis and treatment that improves structure, function, and the body's intrinsic ability to heal. Management begins with taking a standard history and performing a physical exam. If necessary, laboratory and imaging studies are conducted. An osteopathic structural exam is then performed, 
which includes an evaluation and assessment of the following parameters which are beyond the scope of this article: presence of segmental and regional restrictions of motion, thoracic cage immobility, muscle spasms, tenderpoints, viscerosomatic reflexes, Chapman's points, lymphatic obstructions, autonomic abnormalities, and structural irregularities. The patient then receives hands-on manipulation aimed at addressing any specific dysfunctions, in addition to standard-of-care treatment protocols. Finally, reassessment is performed in order to evaluate the efficacy of intervention.

\section{Literature Review}

Our initial inclusion criteria were randomized controlled trials (RCTs) indexed in PubMed studying the efficacy of OMT on adults (> 18 years old) diagnosed with community-acquired pneumonia. We searched PubMed for "osteopath* pneumonia randomized controlled trial", which yielded a return of six articles. Of these results, three fit the scope of the inclusion criteria.

Because of the limited number of RCTs on the efficacy of OMT in pneumonia patients, we expanded our inclusion criteria to include studies on the efficacy of another manual, non-pharmacologic, adjunctive therapy used in the treatment of pneumonia, namely chest physiotherapy. Chest physiotherapy is defined as a set of techniques designed to improve respiratory efficiency by promoting expansion of the lungs, strengthening respiratory muscles, and eliminating secretions from the lungs ${ }^{16}$. Chest physiotherapy includes techniques such as percussion and postural drainage along with coughing and deep breathing exercises, which are indicated for the management of pneumonia ${ }^{17}$. It is clear that the ideal goal of chest physiotherapy is similar to OMT in that both of these manual modalities assist in decreasing the work of breathing and promoting lung expansion. This, it was logical for us to expand our search to include chest physiotherapy.

We then searched PubMed for "physiotherapy pneumonia randomized controlled trial", which yielded a return of 59 articles. Of these results, three additional studies fit the scope of the inclusion criteria.

When combining the two searches, a total of six RCTs fit our criteria for inclusion. These RCTs are described below, summarized and displayed in Figures 3-4:

- In 2010, Noll published Efficacy of osteopathic manipulation as an adjunctive treatment for hospitalized patients with pneumonia: a randomized controlled trial. This was a multi-center randomized controlled trial involving 406 subjects that focused on adjunctive therapies for pneumonia in elderly patients. The treatment group received OMT from an osteopathic specialist, which utilized techniques such as rib raising, doming the diaphragm, thoracic pump, and lymphatic pump. A placebo group received light touch, while a control group received conventional care only. Noll found that when compared to conventional treatment by per-protocol analysis, OMT was associated with decreased length of stay (LOS) and duration of IV antibiotics, as well as a lower incidence of respiratory failure and death. ${ }^{18}$

- In 2000 and 1999, Noll published Benefits of osteopathic manipulative treatment for hospitalized elderly patients with pneumonia and Adjunctive osteopathic manipulative treatment in the elderly hospitalized with pneumonia: a pilot study. These trials reported that adjunctive OMT, when compared to light touch, was associated with decreased LOS and duration of intravenous antibiotics. ${ }^{19,20}$

- In 1997, Bjorkqvist's Bottle-blowing in hospital-treated patients with community acquired-pneumonia compared outcome measures in pneumonia patients stratified to receive an adjunctive protocol for early mobilization, deep breathing, or bubble-blowing. Bubble-blowing was associated with a decreased LOS relative to the early mobilization group. ${ }^{21}$

- In 1985, Britton published Chest physiotherapy in primary pneumonia. This study found no benefit for pneumonia patients undergoing a protocol for postural drainage, percussion, vibration, and external help with breathing. ${ }^{22}$

- In 1978, Graham's Efficacy of chest physiotherapy and intermittent positive-pressure breathing in the resolution of pneumonia found no benefit for pneumonia patients enrolled in a protocol for postural drainage, percussion, vibration, and encouragement of deep breathing and coughing. ${ }^{23}$

\section{Protocol}

The five OMT's described here are a small representation of the osteopathic procedures that can be utilized for a patient with pneumonia when indicated. These techniques focus on improving thoracic cage compliance, improving lymphatic and circulatory flow, and balancing autonomic tone. These techniques are similar to the techniques utilized in the three OMT studies demonstrating efficacy in treating patients with pneumonia.

\section{Muscle Energy to Rib 1 - Exhalation Dysfunction ${ }^{24,25}$}

1. Assess rib 1 motion by placing a first digit on the posterior aspect of the patient's first rib, second digit on the supraclavicular portion of the first rib, and third digit on the infraclavicular portion of the first rib. Palpate for restricted motion or tissue congestion.

2. Patient lies supine on table. Stand on the opposite side of the rib dysfunction (for example, if it is a left Rib 1 Exhalation Dysfunction, stand on the patient's right side and vice versa).

3. Reach the caudad hand under the patient and grasp the rib angle of the dysfunctional rib 1. Apply traction in an inferolateral direction.

4. Place the dorsum of the patient's left wrist on forehead. Then, place a hand over patient's wrist.

5. Have the patient take a deep breath in while moving the rib inferiorly to engage the restrictive barrier.

6. Have the patient hold his or her breath for 3-5 seconds while attempting to lift their head against isometric resistance provided by a hand.

7. Repeat steps 1.5-1.6 three to five times while re-engaging a new restrictive barrier after each repetition.

8. Following the last repetition, a final, passive stretch is performed further into the restrictive barrier.

9. Re-assess rib 1 motion and check for signs of improvement. 


\section{Muscle Energy to Rib 1 - Inhalation Dysfunction ${ }^{24,25}$}

1. Sit at the head of the table while the patient lies supine.

2. Assess for dysfunction using digital palpation of rib 1. Place a thumb on the posterior aspect of the rib, second digit on the supraclavicular portion, and third digit on the infraclavicular region. Feel for restricted motion or local tissue congestion.

3. Monitor the head of the dysfunctional rib in the supraclavicular fossa with a thumb.

4. Flex patient's head forward with the opposite hand until motion is felt at rib 1 in order to relieve the tension of the anterior scalene muscles.

5. Instruct the patient to inhale and exhale deeply. As the patient exhales, move rib 1 inferiorly into the restrictive barrier. Instruct the patient to hold his/her breath in exhalation for 3-5 seconds.

6. While the patient holds his/her breath in exhalation, instruct patient to push his/her head backwards against isometric resistance. This should last for 3-5 seconds while the patient is holding his/her breath.

7. When the patient inhales, resist the natural tendency of the rib to move superiorly with inhalation.

8. Repeat steps $2.5-2.7$ three to five times, while re-engaging a new restrictive barrier with each repetition. After the final repetition, a passive stretch is performed further into the restrictive barrier.

9. Re-asses rib motion to evaluate for any improvement.

\section{Doming the Thoracic Diaphragm ${ }^{24,25}$}

1. Assess thoracic cage motion bilaterally by palpating the rib cage while the patient inhales and exhales.

2. Patient lies supine on the table. Stand on either side of patient. Thumb tips should be placed inferolateral to the xiphoid process and rest along the anterolateral costal margin below rib 7, which corresponds to muscular attachments of the respiratory diaphragm. The remaining digits should rest along the inferolateral border of ribs 8-10.

3. Instruct the patient to "take a deep breath and then breathe all the way out." As the patient exhales, follow the diaphragm by pressing thumbs posterior towards the table.

4. Hold this point on the diaphragm as the patient takes the next deep inhalation. During the next exhalation, a further cephalad motion of the diaphragm is recommended (within a reasonable means and not providing any excessive discomfort to the patient). Continue to monitor the superior movement of the diaphragm.

5. Repeat steps 3.3-3.4 for three to five respiratory cycles, or until the diaphragm domes easily at the end of exhalation.

6. Re-asses by monitoring the diaphragm for improvement in excursion.

\section{Seated Rib Raising 24,25}

1. Assess respiratory motion by palpating the rib cage. In particular, assess specific ribs for individual restrictions that impede the motion of the entire thoracic cage.

2. Begin by having patient seated. Stand facing the patient with one foot behind the other.

3. Instruct patient to cross his or her arms and rest their elbows on your shoulder. Patient may rest his/her head on his/her arms.

4. Reach underneath the arms of the patient. Position finger pads near the costotransverse articulation, at the level of ribs 2-6. The finger pads are used as a fulcrum for extension of the patient's spine.

5. Lean weight onto the back foot and draw patient forward, providing an anterior-lateral traction of the rib angles. Also, extend the patient's spine by shifting center of gravity posteriorly, thereby stretching the intercostal spaces and engaging the restrictive barrier.

6. Hold this position for one second, and then release by allowing your weight to transfer forward to the more anterior foot and the patient to spring back to a more upright position.

7. Move finger pads down one rib level and repeat steps 4.5-4.6. Continue this step-by-step down the rib levels until the rib levels are out of reach (typically around ribs 6-8).

8. Reverse the procedure by working back up the rib cage until reaching rib 2 .

9. Determine success of treatment by reassessing rib motion of previously restricted rib levels.

\section{Thoracic Pump with Respiratory Assist ${ }^{24,25}$}

1. Assess thoracic cage motion bilaterally by palpating the rib cage while the patient inhales and exhales.

2. The patient is positioned supine on table, while the physician stand at the head of the table. The table height should be adjusted to a comfortable height where the hands can fully extend onto patient's pectoral region.

3. Place hands over the patient's pectoral region, with heels of hands just distal to clavicles and thumbs at approximately 45 degrees to sternum.

4. Instruct patient to inhale and exhale deeply. Provide a compressive force downward onto the chest cage. Then, oscillate the degree of compression to produce a pump motion. Continue for approximately one minute or until adequate time passes for proper lymph flow.

5. Respiratory assist is initiated by instructing the patient to inhale deeply and then exhale deeply. During the exhalation phase, follow the chest wall down until exhalation is complete. At the end of exhalation, hold chest wall in place and provide resistance while patient begins inhalation. Follow this step (step 2d) for several cycles of inhalation/expiration (2-6 cycles).

6. During the final inhalation phase, right before the patient has completed a full deep inhalation, rapidly remove hands from patient's chest to allow for a sudden influx of air into the patient's chest.

7. Re-asses for improvements by palpating thoracic motion. 


\section{Representative Results}

First, Muscle Energy Technique targets the Golgi tendon organs, which are stretch receptors located in skeletal muscle. Upon contraction, Golgi tendon organs are stretched, which activates afferent sensory type lb nerves transmitting to the spinal cord. In the central nervous system, type lb nerves synapse upon inhibitory motor neurons, which then provide inhibitory impulses to alpha motor neurons targeting the homonymous muscle group. The end result is relaxation of the initial and synergistic muscles, as well as contraction of antagonistic muscles. This effect is referred to as the Golgi tendon reflex or Inverse Myostatic Reflex. In the application of Muscle Energy Technique to the rib cage, muscles involved in breathing are engaged in isometric resistance. In the case of Rib 1, rib motion can be restricted by pathology related to the scalene muscles. By improving somatic dysfunctions in this respiratory muscle set, Rib 1 moves with greater ease during inspiration and expiration. Treatments that assist thoracic cage motion reduce the impedance of lymphatic flow by respiratory structures, which are located in the thoracic inlet/outlet area. Myofascial restrictions in clavicular region, such as scalene hypertrophy or spasm, can impede the terminal drainage of lymphatic vessels en route to the subclavian veins. Moreover, increased rib cage excursion improves pressure gradients, which further promotes lymph flow.

Second, Doming of the Thoracic Diaphragm Technique involves manipulation of the thoracic diaphragm, which is a principal muscle involved in breathing, blood circulation, lymphatic flow, and other key elements ${ }^{22}$. This technique involves "doming" the muscle to relieve hypertonicity associated with a flattened or dysfunctional state. Doming refers to the method of applying pressure and stretching the muscle in order to return it to a more normal rounded shape; thus, decreasing it's hypertonicity. This technique indirectly engages the inferior surface of the diaphragm and increases its excursion during expiration ${ }^{26}$. Furthermore, the diaphragm assists lymphatic flow by exerting a pump-like propulsion effect on fluid within vessels. Thus, a hindrance in the diaphragm's physical pumping force will limit lymphatic return to circulation. For instance, a hypertonic diaphragm can hamper the lymphatic flow from the cisterna chili, which is a major vessel that lies behind diaphragmatic attachments. The doming of the thoracic diaphragm technique increases excursion, and consequently there will be both optimal lymph flow and return pressure gradients to normal.

Third, Rib Raising Technique augments lymphatic flow by improving respiratory excursion and reducing sympathetic outflow. Excessive autonomic innervation reduces chest wall mobility by generating hypertonicity of the rib cage musculature and increasing intra-abdominal pressure $^{27}$. Since lymphatic flow is dependent on pressure gradients generated by adequate respiratory excursion, excessive sympathetic tone can be a hindrance to lymphatic drainage ${ }^{28}$. Accordingly, this technique addresses this pathology by focusing on sympathetic chain ganglia adjacent to Ribs $2-6^{26}$. Furthermore, in a study comparing the effect of Rib Raising to a light touch control group, there was a significant decrease in the levels of $\alpha$-amylase, an established physiological biomarker of sympathetic activity ${ }^{29}$.

Fourth, Thoracic Pump Technique increases the flow of lymph and other immune cells through a rhythmic, phasic compression of lymphatic vessel walls and regional lymph tissue ${ }^{30}$, especially the thoracic duct. This technique provides a mechanical force to supplement lymphatic drainage into venous circulation, which is primarily useful in states of obstructed or limited drainage of the extracellular compartment. The oscillatory compressive action produces alternating pressure gradients, which enable lymph to flow through its natural channels in a superior direction. 


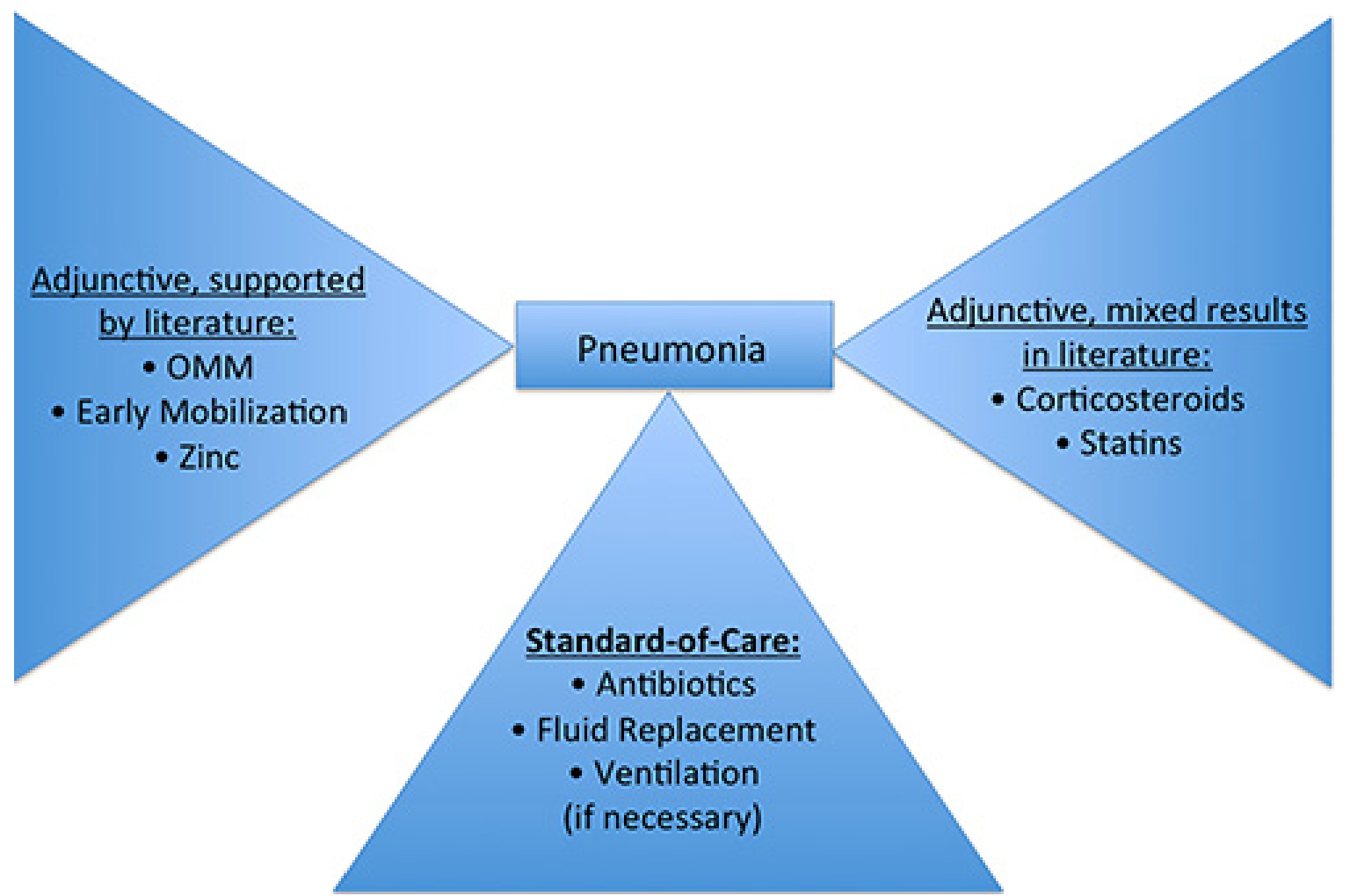

Figure 1. This figure illustrates various components of pneumonia treatment and their efficacy, as supported by current literature ${ }^{4}$.

\section{History}<smiles>C1CC1</smiles>

\section{Physical Exam}<smiles>C=CC</smiles>

Special Tests<smiles>C1CCC1</smiles>

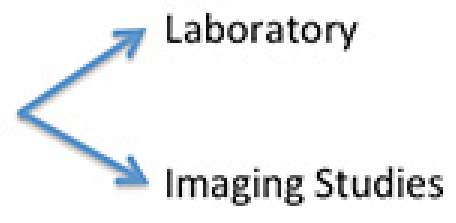

\section{Osteopathic Structural Exam}

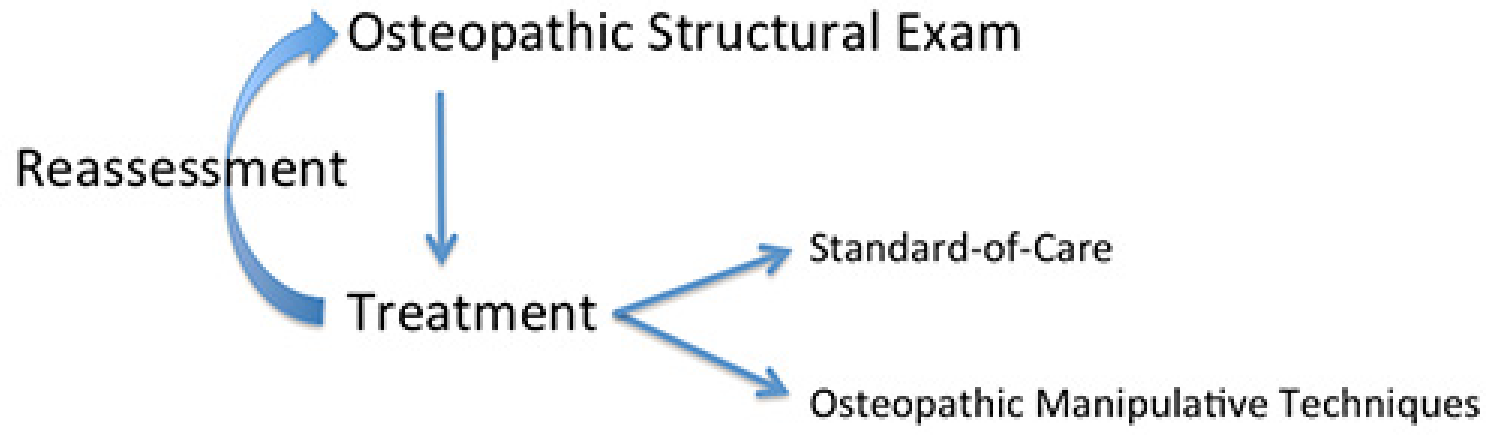

Figure 2. This figure illustrates the step-by-step approach toward patient care utilized by osteopathic physicians. 
10

OMT / Physiotherapy — Light Touch / Conventional Therapy

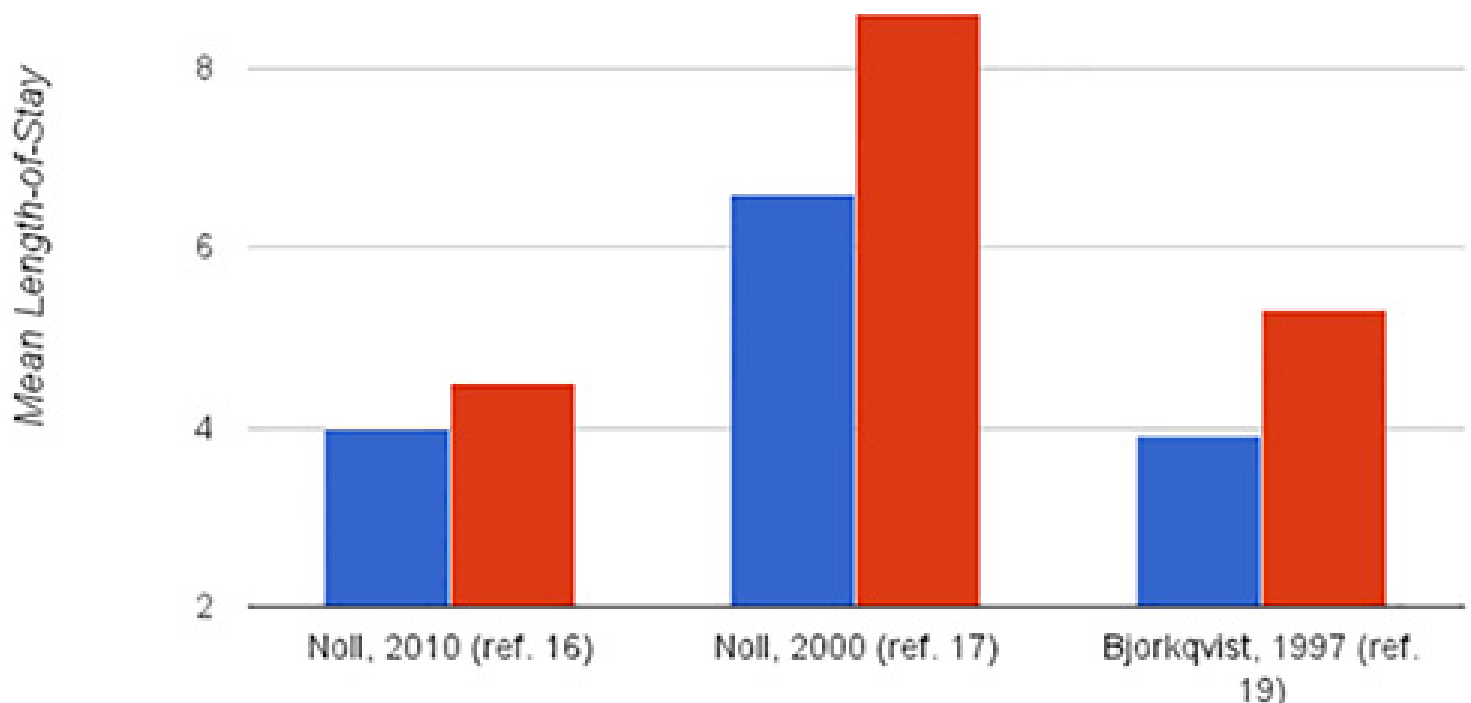

Randomized-Controlled Trial

Figure 3. This figure demonstrates significant findings in RCTs comparing length of stay.

10

OMT / Physiotherapy Light Touch / Conventional Therapy

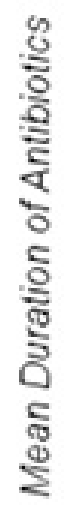

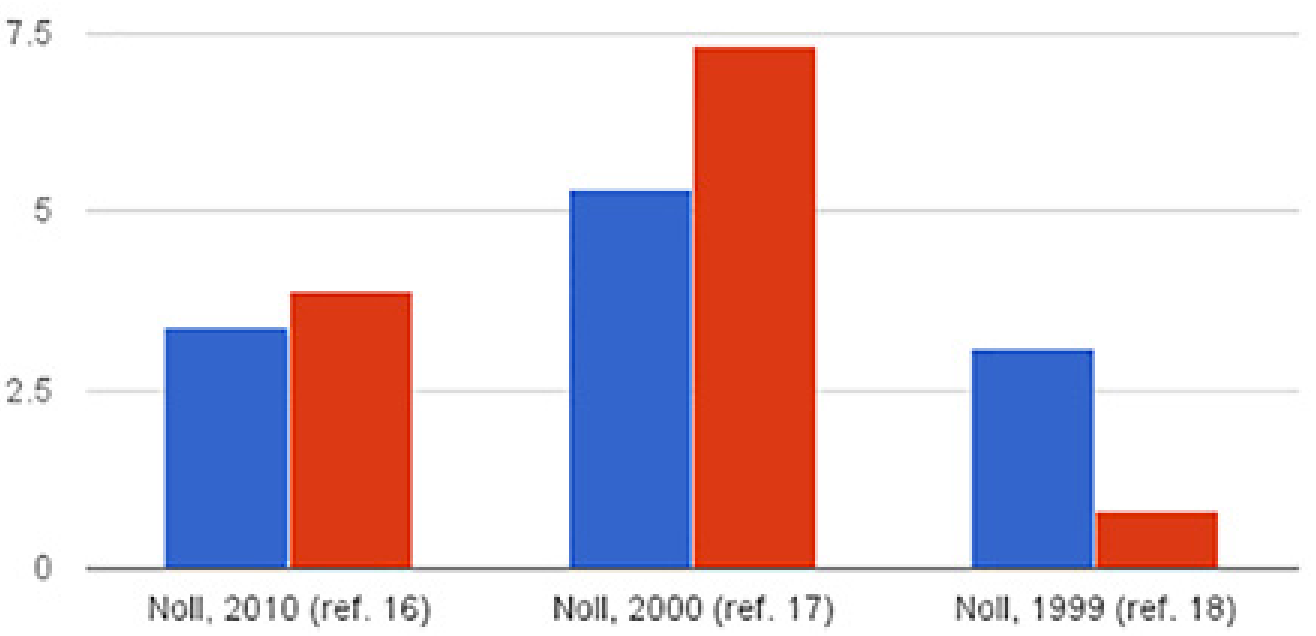

Randomized-Controlled Trial

Figure 4. This figure demonstrates significant findings in RCTs comparing duration of antibiotics. 


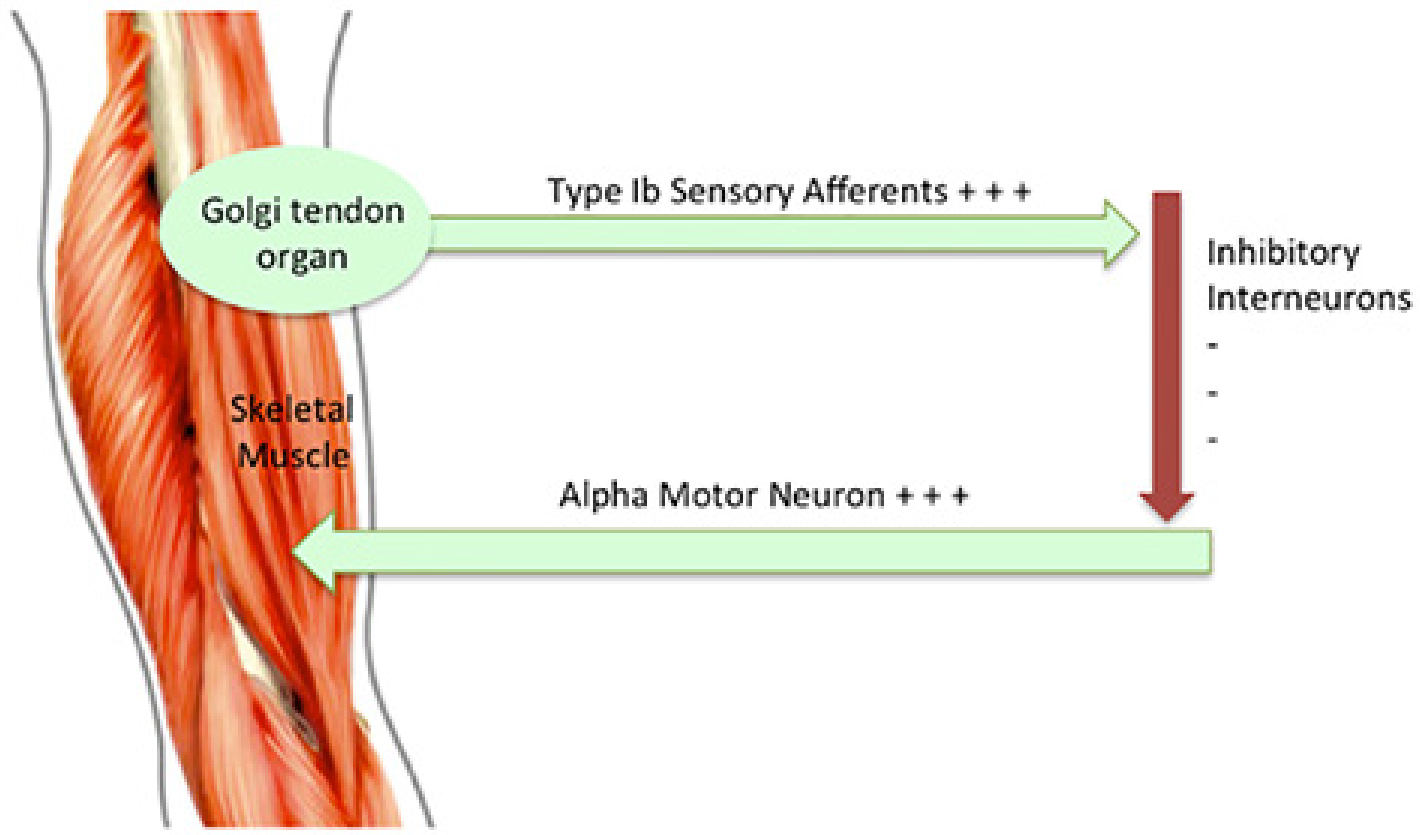

Figure 5. This figure demonstrates the Golgi tendon reflex. When stretched, the Golgi tendon organ is activated and provides feedback to the central nervous system that results in inhibitory impulses being sent to the homonymous muscle.

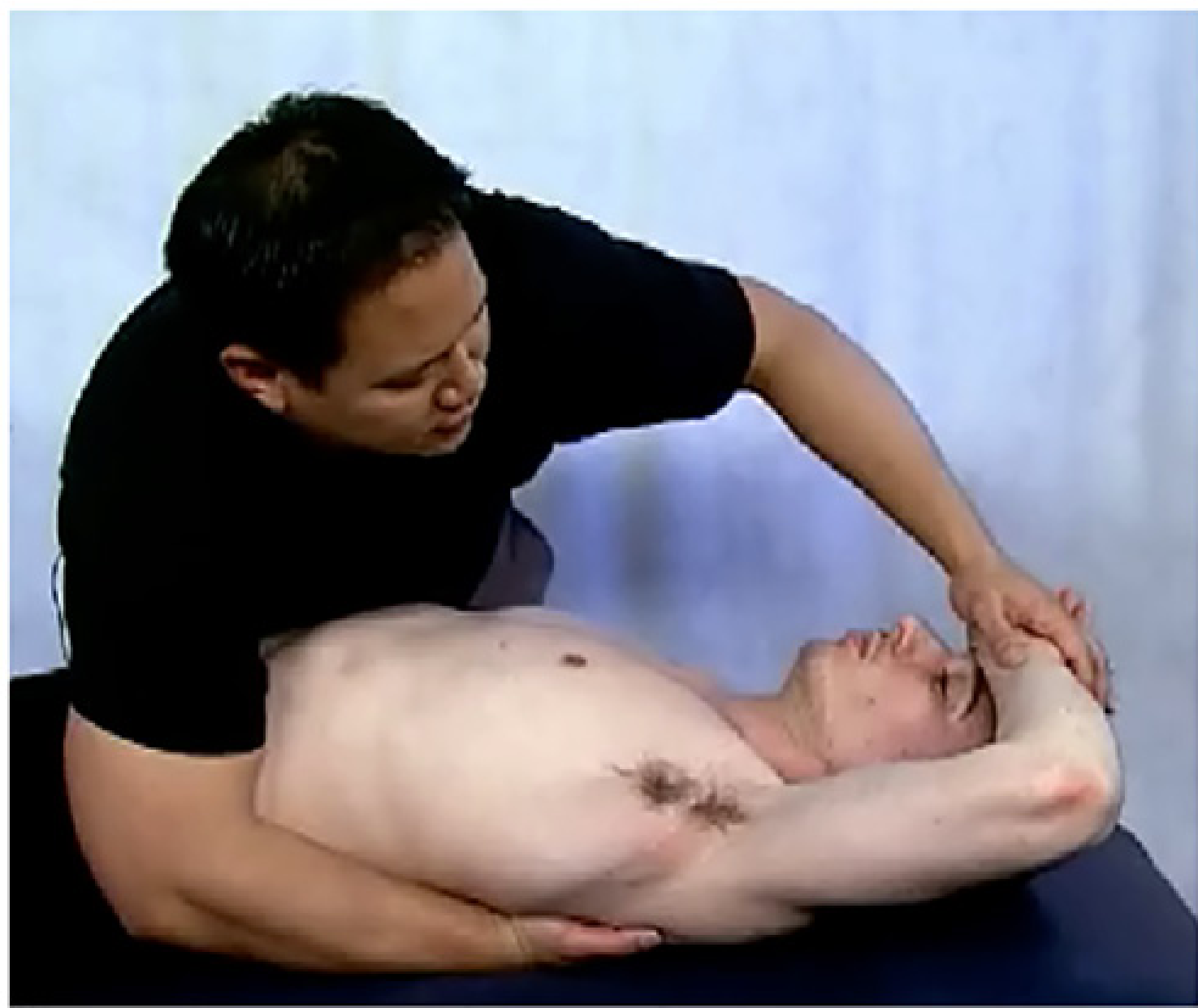

Figure 6. This figure demonstrates the positional set up for Muscle Energy Technique applied to an exhalation dysfunction of Rib 1. 


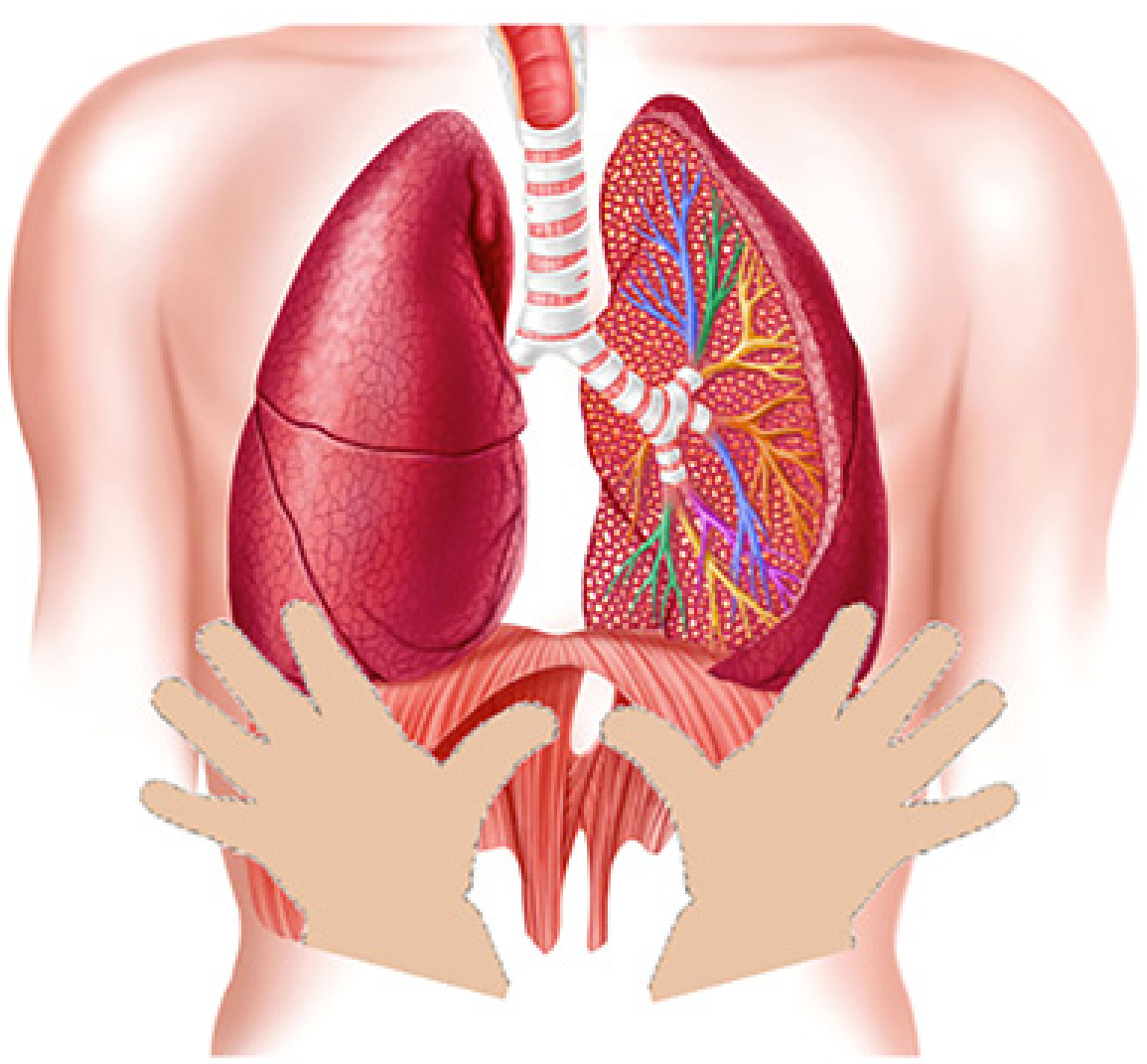

Figure 7. This figure illustrates placement of the thumbs for Doming the Diaphragm Technique, which normalizes the shape of the diaphragm to alleviate abnormal respiratory motions. 


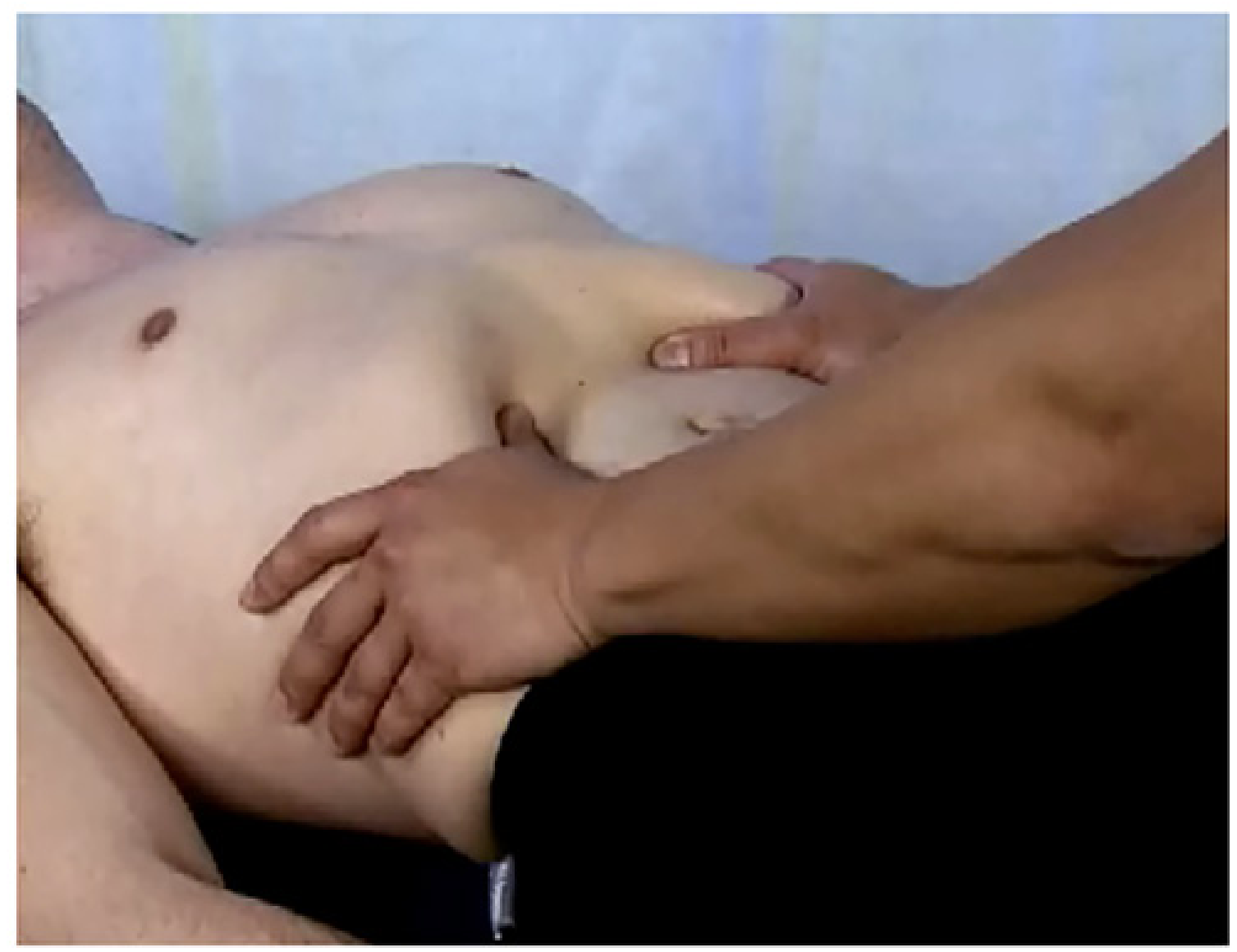

Figure 8. This figure demonstrates the positional set up for Doming The Diaphragm technique. 
jove

Journal of Visualized Experiments

www.jove.com

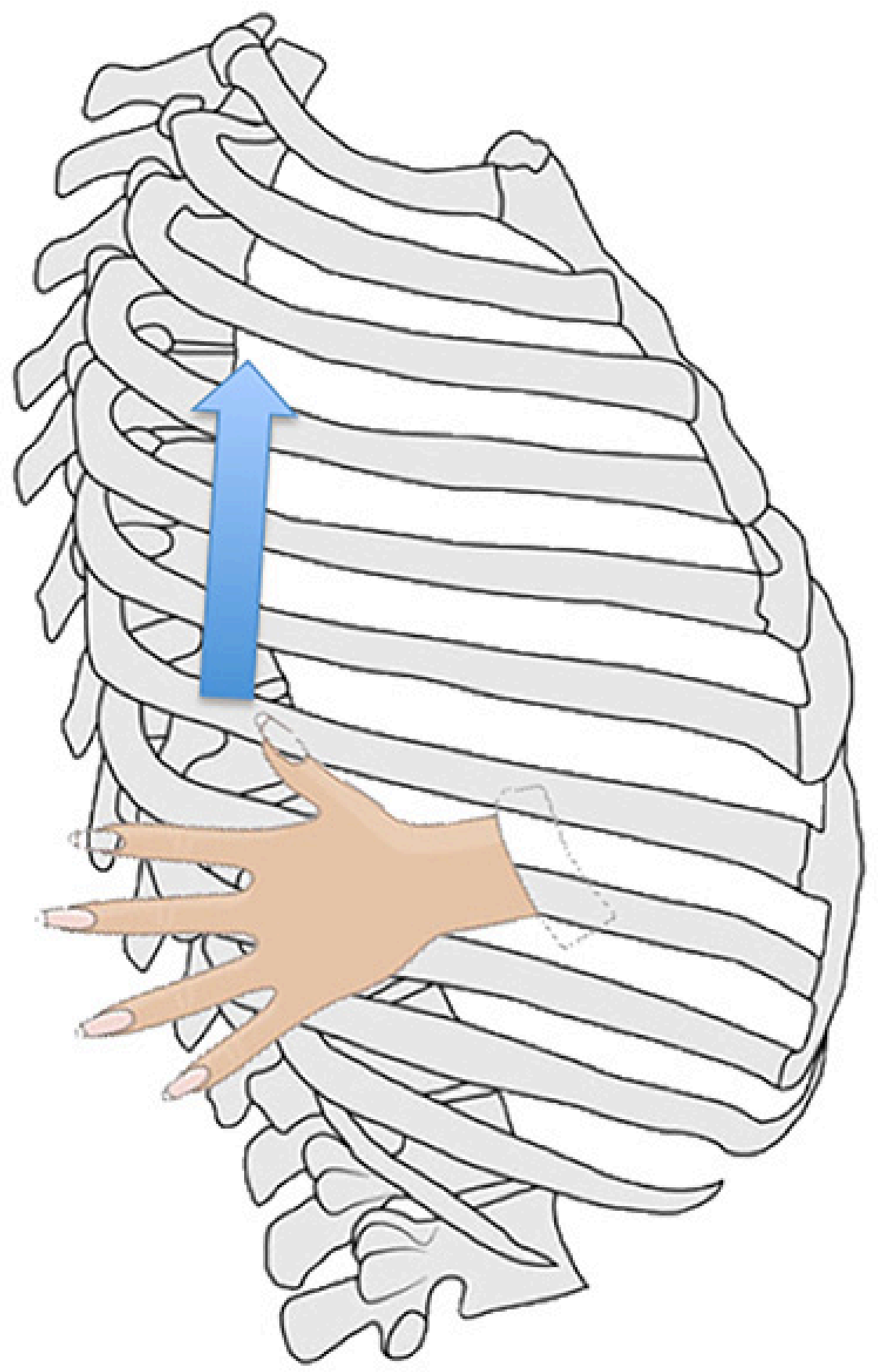

Figure 9. This figure illustrates the Rib Raising Technique, which improves respiratory expansion and alleviates hypertonicity caused by excessive innervation from the sympathetic chain ganglia.

Copyright @ 2014 Creative Commons Attribution-NonCommercial-NoDerivs 3.0 Unsorted

May $2014 \mid 87$ | e50687 | Page 10 of 15

License 


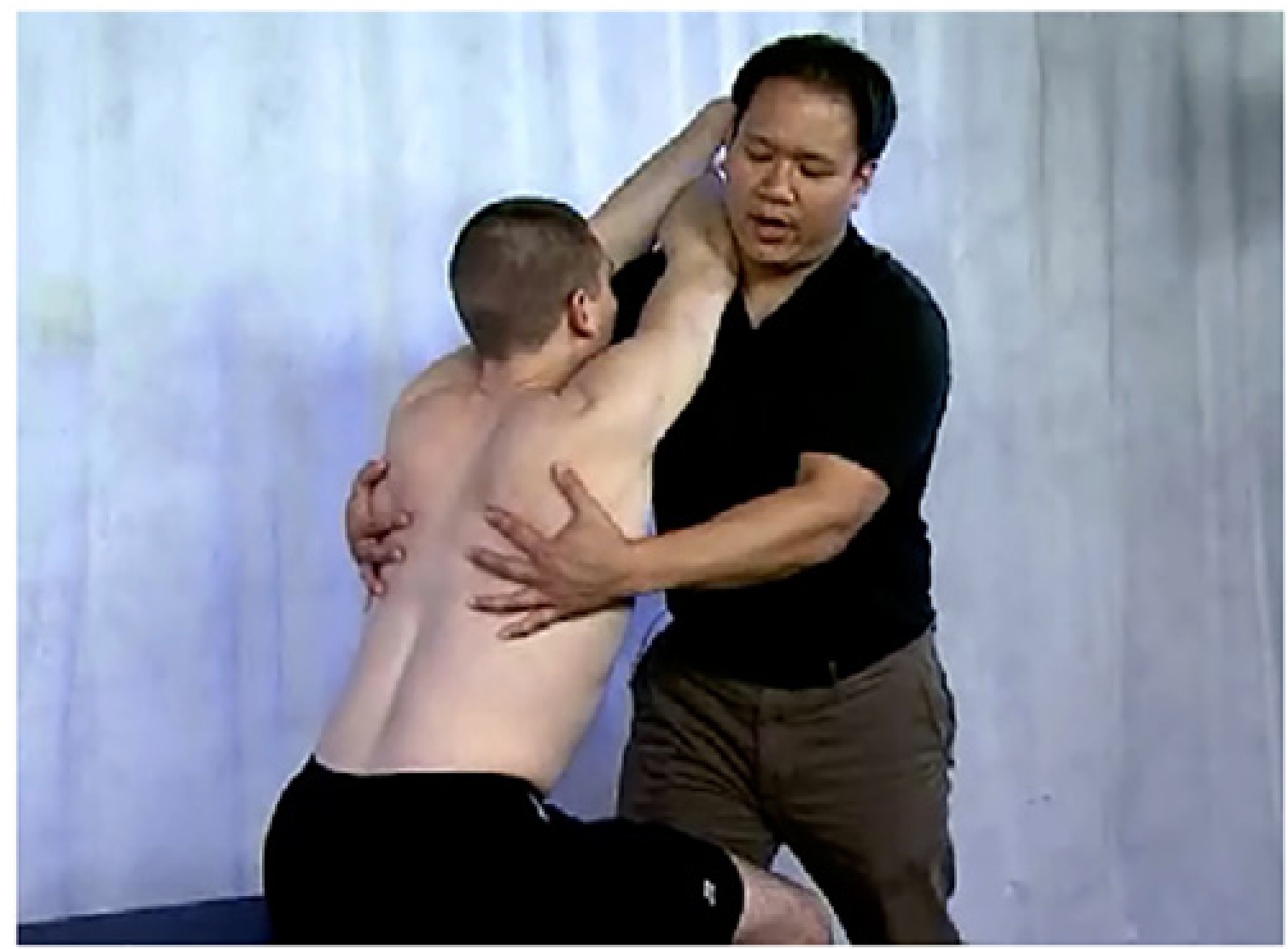

Figure 10. This figure demonstrates the positional set up for Rib Raising Technique. 


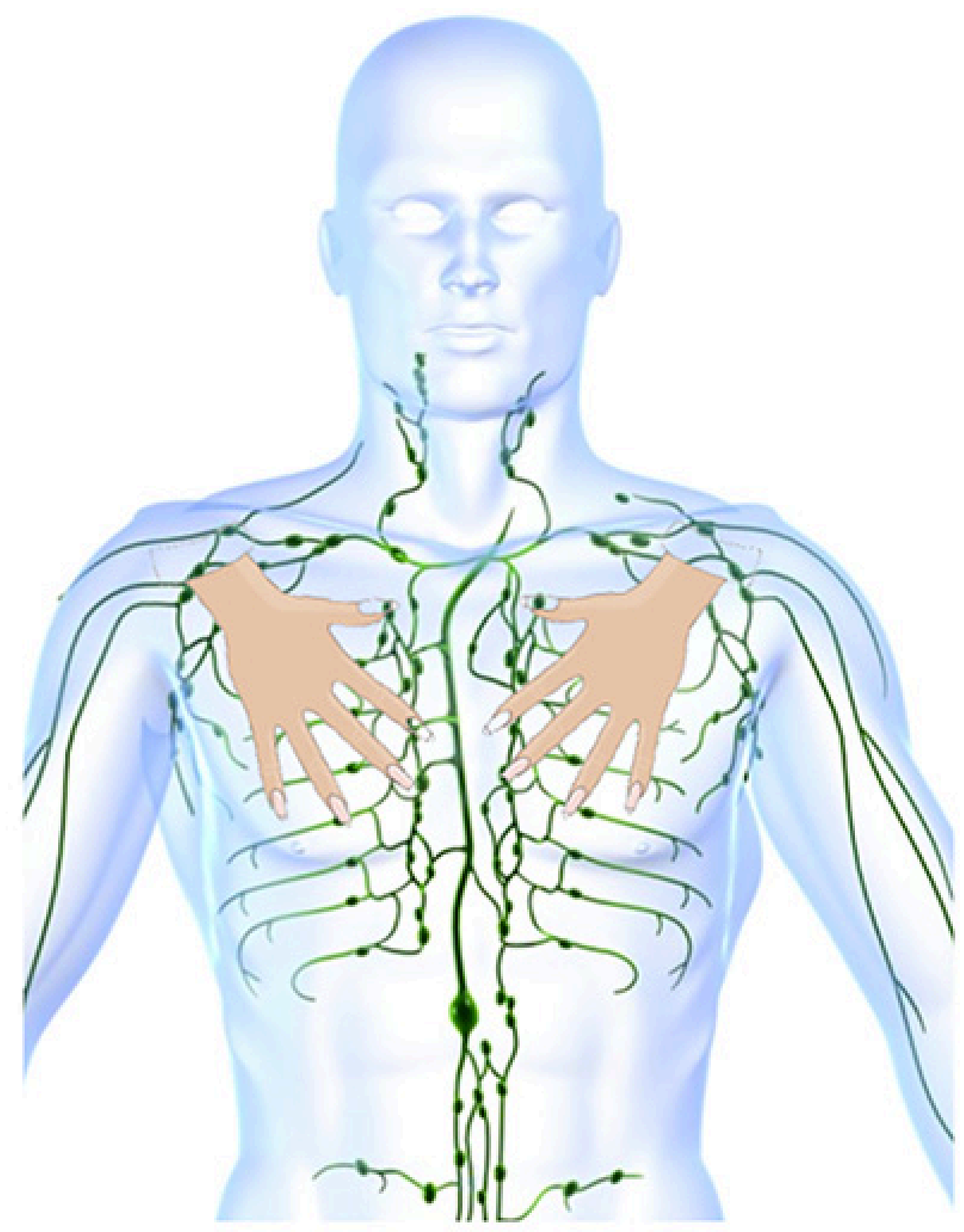

Figure 11. This figure illustrates hand positioning, relative to the lymphatic system, prior to providing downward oscillatory compression in Thoracic Pump Technique. 


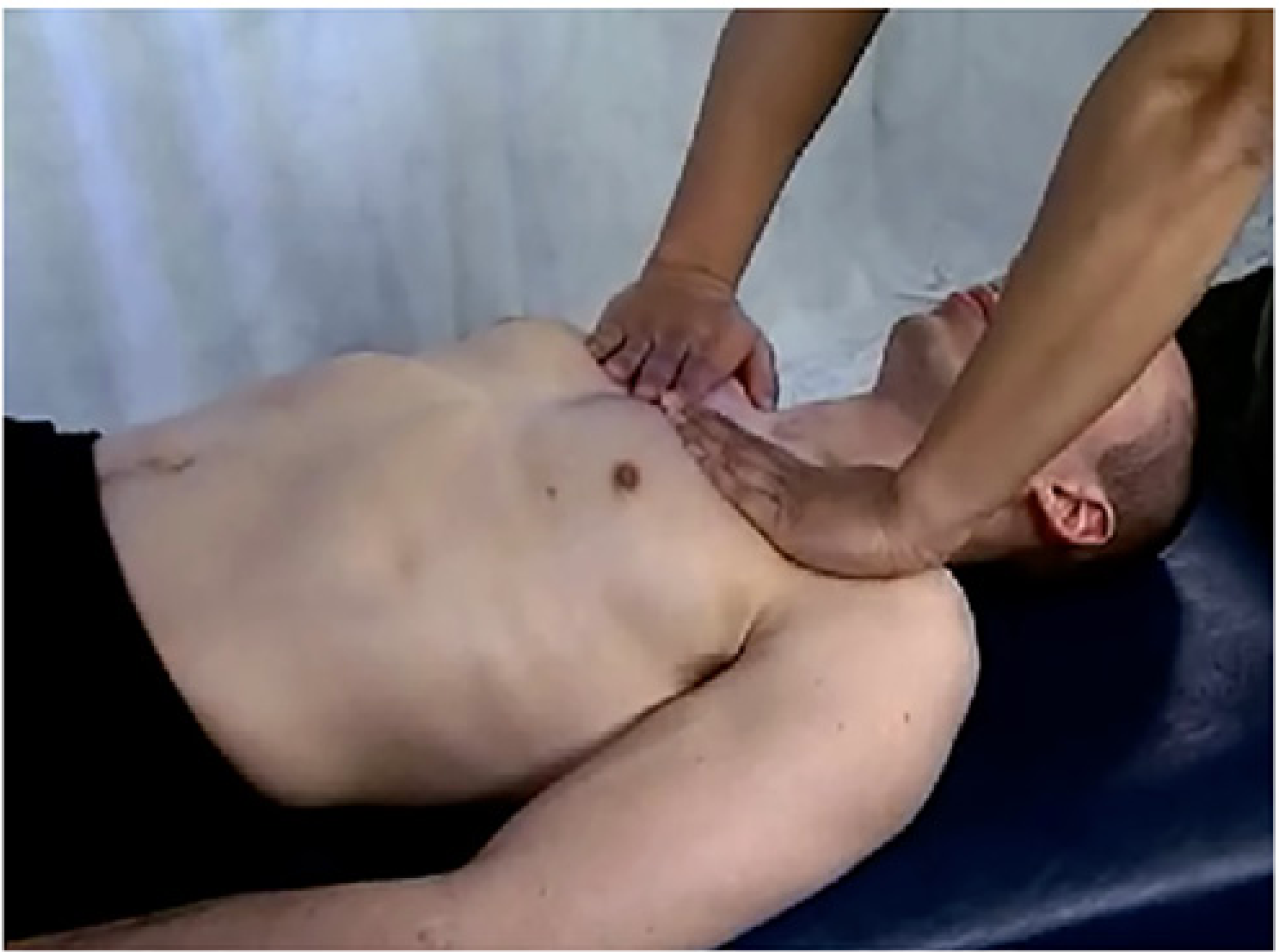

Figure 12. This figure demonstrates the positional set up for Thoracic Pump Technique.

\section{Discussion}

Osteopathic medicine, when applied to pneumonia patients, aids in restoring health by encouraging normal circulation and immunological function. This is achieved in three sequential steps: removal of dysfunctions obstructing drainage, maximization of respiratory function, and augmentation of flow through lymphoid tissue ${ }^{26}$. The roles of the lymphatic system include: balancing the composition of interstitial fluid, transportation of excess substances, distribution of immune cells from lymphoid tissues to systemic circulation, and filtration and removal of foreign material from interstitial fluid.

Literature suggests that techniques targeting lymphatic flow are advantageous for treatment of pneumonia by way of altering pressure differentials in the thoracic diaphragm ${ }^{26}$, improving respiratory function ${ }^{31,32}$, enhancing antibody response ${ }^{7,33}$, reducing edema ${ }^{34}$, activating autonomic-mediated intrinsic lymphatic contractility ${ }^{28}$, and increasing the abundance of white blood cells in peripheral blood ${ }^{8}$. Lymphatic pump techniques have been shown to increase cytokines and chemokines in thoracic and intestinal lymph vessels ${ }^{35}$, while mechanical pressure to certain body regions distant from the location of lymph formation enhances flow into lymphatic systems ${ }^{36}$. In addition, it has been shown that lymphatic OMT augments leukocyte mobilization and flow in dogs and rats, primarily from gut-associated lymphoid tissue ${ }^{37,38,39,40,41}$.

Contraindications to performing OMT include osseous fractures, thrombotic events, certain stages of carcinoma, and bacterial infections with a current temperature over 102 degrees Fahrenheit. Osteopathic manual manipulations should only be performed by trained osteopathic physicians. If the physician has difficulty performing the techniques described, we suggest re-positioning and/or further examination of the patient for additional structural abnormalities.

In practice, clinicians may utilize various modifications of the techniques described. For example, a similar version of the rib raising technique can be performed while the patient is supine. In this arrangement, the physician should use the finger-pads of both hands to contact the patient's rib angles. Utilizing the metacarpophalangeal joints and the treatment table as a fulcrum, the physician should pull laterally by leaning back. This position should be held until a release is felt, then repeat the steps in other areas of the ribs. A modification to the thoracic pump technique involves providing vibratory compressions to the rib cage during patients' exhalation phase.

In conclusion, we believe that OMT is a valuable adjunctive tool in the treatment of pneumonia. We reviewed established protocols by discussing the steps, mechanisms, and physiologies associated with these therapies. Armed with a better understanding of these techniques, we hope that more physicians will opt to incorporate osteopathic principles into infectious disease protocols. 


\section{Disclosures}

Martin Gagne was an Associate Editor at the Journal of Visualized Experiments (JoVE) from 2009 to 2011. The remaining authors have no other disclosures.

\section{Acknowledgements}

The authors would like to thank the entire New York Institute of Technology College of Osteopathic Medicine's Department of Osteopathic Manipulative Medicine. This project is funded by the Department of Osteopathic Manipulative Medicine at the New York Institute of Technology College of Osteopathic Medicine.

\section{References}

1. Xu, J., Kochanek, K., Murphy, S., Tejada-Vera, B. Deaths: Final Data for 2007. National Vital Statistics Reports. 58, (2010).

2. Hall, M.J., DeFrances, C., Williams, S., Golosinskiy, A., Schwartzman A. National Hospital Discharge Survey: 2007 Summary. National Health Statistic Reports. 29, (2010).

3. Centers for Disease Control and Prevention. Prevention and Control of Influenza: Recommendations of the Advisory Committee on Immunization Practices (ACIP), 2007. Morbidity and Mortality Weekly Report. 56, (2007).

4. Haessler, S., Schimmel, J. Managing Community-Acquired Pneumonia During Flu Season. Cleveland Clinical J. of Medicine. 79 67-78 (2012).

5. Noll, D.R., Degenhardt, B.F., Fossum, C., Hensel, K. Clinical and research protocol for osteopathic manipulative treatment of elderly patients with pneumonia. J Am Osteopath Assoc. 108(9). 508-16 (2008).

6. Smith, R.K. One hundred thousand cases of influenza with a death rate of one-fortieth of that officially reported under conventional medical treatment. J. Am. Osteopath. Assoc. 20, 172-175 (1920).

7. Saggio, G., Docimo, S., Pilc, J., Norton, J., Gilliar, W. Impact of osteopathic manipulative treatment on secretory immunoglobulin a levels in a stressed population. J. Am. Osteopath. Assoc. 111, 143-147 (2011).

8. Measel, J.W., Kafity, A.A. The effect of the lymphatic pump on the B and T cells in peripheral blood. J. Am. Osteopath. Assoc. 86, 608 (1986).

9. Allen, T.W., Pence, T.K. The use of the thoracic pump in treatment of lower respiratory tract disease. J. Am. Osteopath. Assoc. 67, 408-411 (1967).

10. Hodge, L.M. Osteopathic Lymphatic pump techniques to enhance immunity and treat pneumonia. Int. J. Osteopath. Med. 15, 13-21 (2011).

11. Hruby, R.J., Hoffman, K.N. Avian influenza: an osteopathic component to treatment. Osteopath. Med. Prim. Care. 9, 10 (2007).

12. Moeckel, E., Mitha, N. Textbook of Pediatric Osteopathy. Churchill Livingstone, 301-304 (2008).

13. Eversteen, J., Baumgardner, D.J., Regnery, A., Banerjee, I. Diagnosis and management of pneumonia and bronchitis in outpatient primary care practices. Primary Care Respiratory Journal. 19(3). 237-241 (2010).

14. Lim, W., van der Eerden, M., Laing, R. Boersma, W., Karalus, N., Town, G., Lewis, S., Macfarlane, J. Defining community acquired pneumonia severity on presentation to hospital: an international derivation and validation study. Thorax. 58 (5) $377-283$ (2003).

15. Fine, M., Auble, T., Yearly, D., Hanusa, B., Weissfeld, L., Singer, D., Coley, C., Marrie, T., Kapoor, W. A prediction rule to identify low-risk patients community-acquired pneumonia. The New England Journal of Medicine. 336. 243-250 (1997).

16. Mitra, P.K. Handbook of Practical Chest Physiotherapy. Jaypee Brothers Medical Publishers Ltd, (2007).

17. Gomella, L.G., Haist, S.A. Chapter 18. Respiratory Care. Clinician's Pocket Reference: The Scut Monkey. 11th ed. New York: McGraw-Hill (2007).

18. Noll D.R., Degenhardt B.F., Morley T.F., Blais F.X., Hortos K.A., Hensel K., Johnson J.C., Pasta D.J., Stoll, S.T. Efficacy of osteopathic manipulation as an adjunctive treatment for hospitalized patients with pneumonia: a randomized controlled trial. Osteopath. Med. Prim. Care. 19, 2 (2010).

19. Noll, D.R., Shores, J.H., Gamber, R.G., Herron, K.M., Swift Jr., J. Benefits of osteopathic manipulative treatment for hospitalized elderly patients with pneumonia. J Am Osteopath Assoc. 100 (12) 776-82 (2000).

20. Noll, D.R., Shores, J., Bryman P.N., Masterson, E.V. Adjunctive osteopathic manipulative treatment in the elderly hospitalized with pneumonia: a pilot study. J Am Osteopath Assoc. 99 (3) 143-152 (1999).

21. Bjorkqvist, M., Wiberg, B., Bodin, L., Barany, M., Holmberg, H. Bottle-blowing in hospital-treated patients with community-acquired pneumonia. Scand J Infect Dis. 29 (1) 77-82 (1997).

22. Britton, S., Bejstedt, M., Vedin, L. Chest Physiotherapy in Primary Pneumonia. Br Med J (Clin Res Ed). 290 (6483) 1703-4 (1985).

23. Graham, W.G., Bradley, D.A. Efficacy of chest physiotherapy and intermittent positive-pressure breathing in the resolution of pneumonia. $N$ Eng J Med. 299 (12) 624-7 (1978).

24. DiGiovanna, E., Schiowitz, S., Dowling, D. An Osteopathic Approach to Diagnosis and Treatment. Lippincott Williams \& Wilkins, (2004).

25. Chila, A. Foundations of Osteopathic Medicine. Lippincott Williams \& Wilkins. 797-803. (2011).

26. Ward, R.W. Foundations for Osteopathic Medicine. Lippincott Williams \& Wilkins. 393-1077 (2003).

27. Pelosi, P. Quintel, M., Malbrain, ML. Effect of intra-abdominal pressure on respiratory mechanics. Acta Clin Belg Suppl. 1. 78-88 (2007).

28. Degenhardt, B.F., Kuchera, M.L. Update on osteopathic medical concepts and the lymphatic system. J. Am. Osteopath. Assoc. 96, 97-100 (1996).

29. Henderson, A.T., Fisher, J.F., Blair, J., Shea, C., Li, T.S., Bridges, K.G. Effects of rib raising on the autonomic nervous system: a pilot study using noninvasive biomarkers. J. Am. Osteopath Assoc. 6, 324-330 (2010).

30. Downey, H.F., Durgam, P., Williams Jr., A.G., Rajmane, A., King, H.H., Stoll, S.T. Lymph flow in the thoracic duct of conscious dogs during lymphatic pump treatment, exercise, and expansion of extracellular fluid volume. Lymphat. Res. Biol. 6, 3-13 (2008).

31. Beicastro, M.R., Backes, C.R., Chila, A.G. Bronchiolitis: A pilot study of osteopathic manipulative treatment, bronchodilators, and other therapy. J. Am. Osteopath. Assoc. 83, 672-676 (1984). 
32. Sleszynski, S.L., Kelso, A.F. Comparison of thoracic manipulation with incentive spirometry in preventing postoperative atelectasis. J. Am. Osteopath. Assoc. 93, 834-845 (1993).

33. Jackson, K.M. Effect of lymphatic and splenic pump techniques on the antibody response to hepatitis B vaccine: a pilot study. J. Am. Osteopath. Assoc. 98, 155-160 (1998).

34. Härén, K., Backman, C., Wiberg, M., Scand, J. Effect of manual lymph drainage as described by Vodder on oedema of the hand after fracture of the distal radius: a prospective clinical study. Plast. Reconstr. Surg. Hand Surg. 34, 367-372 (2000).

35. Schander, A., Downey, H.F., Hodge, L.M. Lymphatic pump manipulation mobilizes inflammatory mediators into lymphatic circulation. J. Exp. Biol. Med. 237 58-63 (2012).

36. Dery, M., Winterson, B., Yonuschot, G. The effect of lymphatic pump manipulation on healthy and injured rat. Lymphology. 33, 58-61 (2000).

37. Knott, E.M., Tune, J.D., Stoll, S.T., Downey, H.F. Increased lymphatic flow in the thoracic duct during manipulative intervention. J. Am. Osteopath. Assoc. 105, 447-456 (2005).

38. Hodge, L.M., King, H.H., Williams Jr., A.G., Reder, S.J., Belavadi, T.J., Simecka, J.W., et al. Abdominal lymphatic pump treatment increases leukocyte count and flux in thoracic duct lymph. Lymphat. Res. Biol. 5, 127-133 (2007).

39. Hodge, L.M., Bearden, M.K., Schander, A., Huff, J.B., Williams Jr., A., King, H.H., Downey, H.F. Lymphatic pump treatment mobilizes leukocytes from the gut associated lymphoid tissue into lymph. Lymphat. Res. Biol. 8, 103-110 (2010).

40. Prajapati, P. Lymphatic pump treatment increases thoracic duct lymph flow in conscious dogs with edema due to constriction of the inferior vena cava. Lymphat. Res. Biol. 8, 149-154 (2010).

41. Huff, J.B., Schander, A., Downey, H.F., Hodge, L.M. Lymphatic pump treatment augments lymphatic flux of lymphocytes in rats. Lymphat. Res. Biol. 8, 183-187 (2010). 